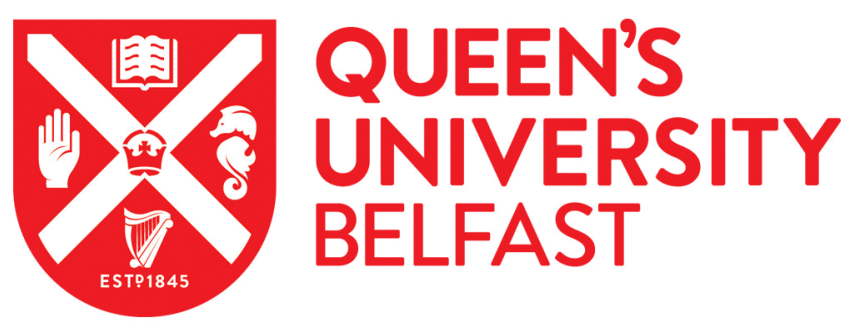

\title{
Search for Dust Emission from (24) Themis Using the Gemini-North Observatory
}

Hsieh, H. H., Kim, Y., Fitzsimmons, A., \& Sykes, M. V. (2018). Search for Dust Emission from (24) Themis Using the Gemini-North Observatory. Publications of the Astronomical Society of the Pacific.

https://doi.org/10.1088/1538-3873/aaca8b

Published in:

Publications of the Astronomical Society of the Pacific

Document Version:

Peer reviewed version

Queen's University Belfast - Research Portal:

Link to publication record in Queen's University Belfast Research Portal

Publisher rights

(c) 2018 The Astronomical Society of the Pacific. This work is made available online in accordance with the publisher's policies. Please refer to any applicable terms of use of the publisher.

\section{General rights}

Copyright for the publications made accessible via the Queen's University Belfast Research Portal is retained by the author(s) and / or other copyright owners and it is a condition of accessing these publications that users recognise and abide by the legal requirements associated with these rights.

Take down policy

The Research Portal is Queen's institutional repository that provides access to Queen's research output. Every effort has been made to ensure that content in the Research Portal does not infringe any person's rights, or applicable UK laws. If you discover content in the Research Portal that you believe breaches copyright or violates any law, please contact openaccess@qub.ac.uk. 
DRAFT VERSION 2018-06-06

Typeset using $\mathrm{LAT}_{\mathrm{E}} \mathrm{X}$ twocolumn style in AASTeX62

\author{
Search for Dust Emission from (24) Themis Using the Gemini-North Telescope* \\ Henry H. Hsieh, ${ }^{1,2}$ Yoonyoung Kim, ${ }^{3}$ Alan Fitzsimmons, ${ }^{4}$ and Mark V. Sykes ${ }^{1}$ \\ ${ }^{1}$ Planetary Science Institute, 1700 East Fort Lowell Rd., Suite 106, Tucson, AZ 85719, USA \\ ${ }^{2}$ Institute of Astronomy and Astrophysics, Academia Sinica, P.O. Box 23-141, Taipei 10617, Taiwan \\ ${ }^{3}$ Department of Physics and Astronomy, Seoul National University, Gwanak, Seoul 151-742, Republic of Korea \\ ${ }^{4}$ Astrophysics Research Centre, Queens University Belfast, Belfast BT7 1NN, United Kingdom
}

(Received 2018 April 27; Revised 2018 June 1; Accepted 2018 June 4)

\title{
Submitted to PASP
}

\begin{abstract}
We report the results of a search for a dust trail aligned with the orbit plane of the large main-belt asteroid (24) Themis, which has been reported to have water ice frost on its surface. Observations were obtained with the GMOS instrument on the Gemini-North Observatory in imaging mode, where we used a chip gap to block much of the light from the asteroid, allowing us to take long exposures while avoiding saturation by the object. No dust trail is detected within $2^{\prime}$ of Themis to a $3-\sigma$ limiting surface brightness magnitude of $\Sigma_{\mathrm{lim}} \sim 29.7 \mathrm{mag} \operatorname{arcsec}^{-2}$, as measured along the expected direction of the dust trail. Detailed consideration of dust ejection physics indicates that particles large enough to form a detectable dust trail were unlikely to be ejected as a result of sublimation from an object as large as Themis. We nonetheless demonstrate that our observations would have been capable of detecting faint dust emission as close as $20^{\prime \prime}$ from the object, even in a crowded star field. This approach could be used to conduct future searches for sublimation-generated dust emission from Themis or other large asteroids closer to perihelion than was done in this work. It would also be useful for deep imaging of collisionally generated dust emission from large asteroids at times when the visibility of dust features are expected to be maximized, such as during orbit plane crossings, during close approaches to the Earth, or following detected impact events.
\end{abstract}

Keywords: minor planets, asteroids: general - minor planets, asteroids: individual, (24) Themis

\section{INTRODUCTION}

\subsection{Background}

Dust emission from solar system bodies is most commonly associated with comets from the outer solar system, but there are situations in which asteroids in the inner solar system may be expected and, in some cases, have even been observed to also eject dust. Collisional erosion of asteroid surfaces is one means by which asteroids can be sources of dust and debris in their orbital

Corresponding author: Henry Hsieh

hhsieh@psi.edu

* This is an accepted manuscript version of an article accepted for publication in Publications of the Astronomical Society of the Pacific. Nether the Astronomical Society of the Pacific nor IOP Publishing Ltd is responsible for any errors or omissions in this version of the manuscript or any version derived from it. vicinity. Because impact velocities in the main asteroid belt are $\sim 5 \mathrm{~km} \mathrm{~s}^{-1}$ (Bottke et al. 1994), significant energy is transferred to ejecta during an impact event, a significant fraction of which will escape the gravitational field of an asteroid. Dust is also produced in the catastrophic disruptions of parent asteroids believed to create asteroid families (Nesvorný et al. 2003, 2006, 2008), taking the form of dust bands that have been identified in data obtained by the Infrared Astronomical Satellite (Low et al. 1984; Dermott et al. 1984; Sykes 1990).

Asteroids on cometary orbits (ACOs), which appear inactive but have comet-like orbits (as defined by having Tisserand parameter values of $T_{J}<3$; Kresák 1972), are often believed to be dormant comets (e.g., Fernández et al. 2001, 2005; Licandro et al. 2006, 2008), and so may have the potential to exhibit new dust emission due to reactivation or residual dust emission from earlier, more 
active periods (e.g., Mommert et al. 2014). Active asteroids have asteroid-like orbits (as defined by having $T_{J}>3$ ) but exhibit comet-like dust emission due to various mechanisms (Jewitt et al. 2015b), and consist of disrupted asteroids, which exhibit activity due to processes such as impact disruptions or rotational destabilization (cf. Hsieh et al. 2012a), and main-belt comets (MBCs), which exhibit activity determined to be due to the sublimation of volatile ice (Hsieh \& Jewitt 2006; Snodgrass et al. 2017).

While most currently known active asteroids are relatively small (effective nucleus radii of $r_{n} \lesssim 5 \mathrm{~km}$; references in Jewitt et al. 2015b), two large disrupted asteroids have been observed, namely (596) Scheila and (493) Griseldis, which have $r_{n}=79.9 \mathrm{~km}$ and $r_{n}=20.8 \mathrm{~km}$, respectively (Mainzer et al. 2016). The dust emission observed for each object is believed to have been caused by a non-catastrophic impact on each body (Jewitt et al. 2011; Bodewits et al. 2011; Ishiguro et al. 2011a; Tholen et al. 2015). Scheila and Griseldis were both quite bright when they were discovered to be active, with apparent $V$-band magnitudes of $m_{V}=14.3 \mathrm{mag}$ and $m_{V}=15.7 \mathrm{mag}$ (according to the JPL Horizons online ephemeris generator ${ }^{1}$ ), respectively. Main-belt dwarf planet (1) Ceres $\left(r_{n}=467.6 \mathrm{~km}\right.$; Carry et al. 2008), is sometimes considered an active asteroid due to the fact that it has been observed to exhibit water vapor outgassing (Küppers et al. 2014), but visible dust emission has never been observed from this body. No large $\left(r_{n}>5 \mathrm{~km}\right)$ MBCs or MBC candidates seen to exhibit observable dust emission are currently known.

\subsection{Asteroid Ice}

Volatile material is known to have existed and to still exist in asteroids. Meteorites linked to the asteroid belt contain aqueously altered minerals, indicating that liquid water was once present in main belt objects (e.g., Hiroi et al. 1996; Burbine 1998; Keil 2000). Even long before the discovery of water vapor outgassing discussed above (Section 1.1), Ceres was thought to possess present-day surface water ice (Lebofsky et al. 1981; Vernazza et al. 2005), which has now been directly detected by Dawn (Combe et al. 2016). Spectroscopic evidence of water ice frost has also been reported for main-belt asteroid (24) Themis (Rivkin \& Emery 2010; Campins et al. 2010), fellow Themis family asteroid (90) Antiope (Hargrove et al. 2015), and other asteroids (Takir \& Emery 2012). Volatile material in main-belt asteroids is a subject of great interest in astrobiology, given dynamical studies indicating that objects from the region

1 https://ssd.jpl.nasa.gov/horizons.cgi of the solar system occupied by the present-day main asteroid belt, or at least similar to objects currently occupying the present-day main belt, could have played a significant role in the primordial delivery of water to the terrestrial planets (Morbidelli et al. 2000; Raymond et al. 2004; Raymond \& Izidoro 2017; O'Brien et al. 2006, 2018, and references within).

The Themis asteroid family has come to be of particular interest in studies of asteroid ice in recent years. Along with the spectroscopic detections of surface ice on Themis and Antiope discussed above, at least three MBCs are likely members of the family (Hsieh et al. 2004, 2011a, 2012b). Since asteroid family members are believed to be compositionally similar, this evidence of probable ice on multiple Themis family members suggests that ice could be widespread in the family.

While the presence of ice on MBCs is inferred indirectly from their dust emission activity, no spectroscopic detections of water ice on a MBC exhibiting visible dust emission have ever been made. The nuclei of the known MBCs are simply too small $\left(r_{n} \ll 5 \mathrm{~km}\right)$ and faint $\left(m_{V} \gg 20\right.$ mag when inactive) to obtain spectroscopic observations sensitive enough to detect any water ice on their surfaces that may be present. Conversely, detectable cometary activity has never been observed for Themis and Antiope despite the reported spectroscopic detections of water ice on their surfaces. Attempts to directly detect sublimation from Themis have been made but have been unsuccessful thus far (Jewitt \& GuilbertLepoutre 2012; McKay et al. 2017). However, given that MBCs exhibit visible dust emission without exhibiting detectable outgassing (see references in Snodgrass et al. 2017), the same could be true for Themis. A major challenge to searching for dust emission from Themis, though, is that it is difficult to efficiently obtain useful deep-imaging observations of such a bright object with a large telescope without quickly saturating the detector and accumulating intractable levels of scattered light.

Photometric analysis (i.e., searching for photometric enhancements that could indicate the presence of unresolved ejected material) is an alternative method of detecting dust emission activity from an object (e.g., Bus et al. 1988; Tholen et al. 1988; Cikota et al. 2014; Hsieh \& Sheppard 2015). Use of this method, however, requires that the amount of ejected dust contained within the seeing disk of an object has a total scattering surface area comprising a detectable fraction of the scattering surface area of the object itself. For very large objects like Themis, this requirement may represent an implausibly large amount of material to be ejected. Higher quality observations with greater photometric precision could improve sensitivity to smaller amounts of ejected 
material, but even then, this method would be limited by the precision of the predictions of Themis's magnitude when inactive, as well as the need for photometric excesses to exceed photometric uncertainties due to rotational variations and aspect angle effects in order to be conclusively attributable to ejected material.

In this paper, we present a search for visible dust emission associated with Themis, where our observations and data reduction techniques were specially designed to address the challenges of performing deep imaging of bright asteroids using relatively simple observing techniques (i.e., suitable for queue mode observing) and instrumentation. While we did not detect any dust emission with these particular observations, we suggest that this approach could be used in the future to perform additional searches for faint activity associated with Themis at different points in its orbit, and also faint activity associated with other bright asteroids in general.

\section{OBSERVATIONS}

A primary consideration in the design of our observations to search for dust emission from Themis was the asteroid's extreme brightness, meaning that any long exposure by a large (8-m-class) telescope would quickly saturate. Bleeding of excess charge, as well as extensive scattered light, would then obscure any faint dust features from view, particularly if the largest column density of dust particles is close to the object. On the other hand, a long series of extremely short exposures to avoid saturating individual exposures would be inefficient both to obtain and process. To address these challenges, we devised a method to observe Themis using one of the chip gaps of a multi-element mosaic imager to block the majority of the light from the asteroid, allowing us to take much longer exposures than would be feasible otherwise.

Another consideration in the design of our observations was timing. While dust emission activity due to sublimation is likely to be strongest near perihelion, or perhaps shortly afterwards (cf. Hsieh et al. 2011b), dust ejected early in an object's active period is expected to be concentrated near the source object. This is because shortly after activity begins, ejected particles do not have time to travel very far, unless they are very small (e.g., $\mu \mathrm{m}$-scale or smaller), in which case they disperse quickly and rapidly become too diffuse to be visible at large distances from the object. For an extremely bright asteroid like Themis, though, dust features close to the object are difficult to detect due to interference from scattered light from the asteroid itself.

At longer times after the start of dust emission, large dust particles (e.g., mm-scale or larger, which disperse much more slowly in response to solar radiation pressure and Keplerian shear) can form a dust trail that is relatively long-lasting and extends to large distances from the source object, avoiding the large amount of scattered light expected near the object. These trails are commonly seen in associated with short-period comets (Sykes \& Walker 1992; Reach et al. 2007), and are the type of dust trail that we aimed to search for with the observations of Themis presented here.

We obtained queue observations of Themis on 2015 June 9 and 2015 June 12 (Table 1) using the $8.1 \mathrm{~m}$ Gemini North telescope (Program GN-2015A-FT-19) on Mauna Kea in Hawaii. At the time of our observations, Themis was at a true anomaly of $\nu \sim 117^{\circ}$, similar to the orbit positions at which other MBCs have been observed to show long, faint dust trails detectable with observations similar to our planned Gemini observations of Themis (i.e., 238P/Read and 324P/La Sagra; Hsieh et al. 2009; Hsieh 2014).

Observations were made using the imaging mode of the Gemini Multi-Object Spectrograph (GMOS; image scale of $0.1454^{\prime \prime}$ pixel $^{-1}$; Hook et al. 2004) and a Sloan $r^{\prime}$ filter. Using accurate knowledge of the position and nonsidereal rate of motion of Themis from Horizons ${ }^{2}$, we were able to design our observations such that minimal effort would be required on the part of queue observers to maintain the object's position in the GMOS chip gap once the telescope was positioned properly at the start of observations.

Later, this same accurate knowledge of Themis's position and non-sidereal rate of motion allowed us to accurately combine our data even though Themis itself was not directly imaged by our observations. Using this technique, assuming 1".0 (FWHM) seeing conditions in $r^{\prime}$-band, placing Themis in the 2". 8 chip gap between the GMOS e2V DD chips would allow us to avoid $\gtrsim 95 \%$ of the flux from the asteroid (Diego 1985), minimizing the potential for saturation, with the remaining flux in the wings of the object's surface brightness profile reaching the detector on either side of the chip gap. Observations comprising one hour of total effective exposure time were obtained on each night, giving us a total of two hours of total on-source integration time.

\section{DATA REDUCTION}

We performed standard bias subtraction and flat-field reduction for all data using sky flats constructed from dithered science images. Flux calibration was conducted using field star photometry from the Pan-STARRS1 survey catalog (Tonry et al. 2012; Schlafly et al. 2012; Mag-

\footnotetext{
${ }^{2}$ http://ssd.jpl.nasa.gov/horizons.cgi
} 

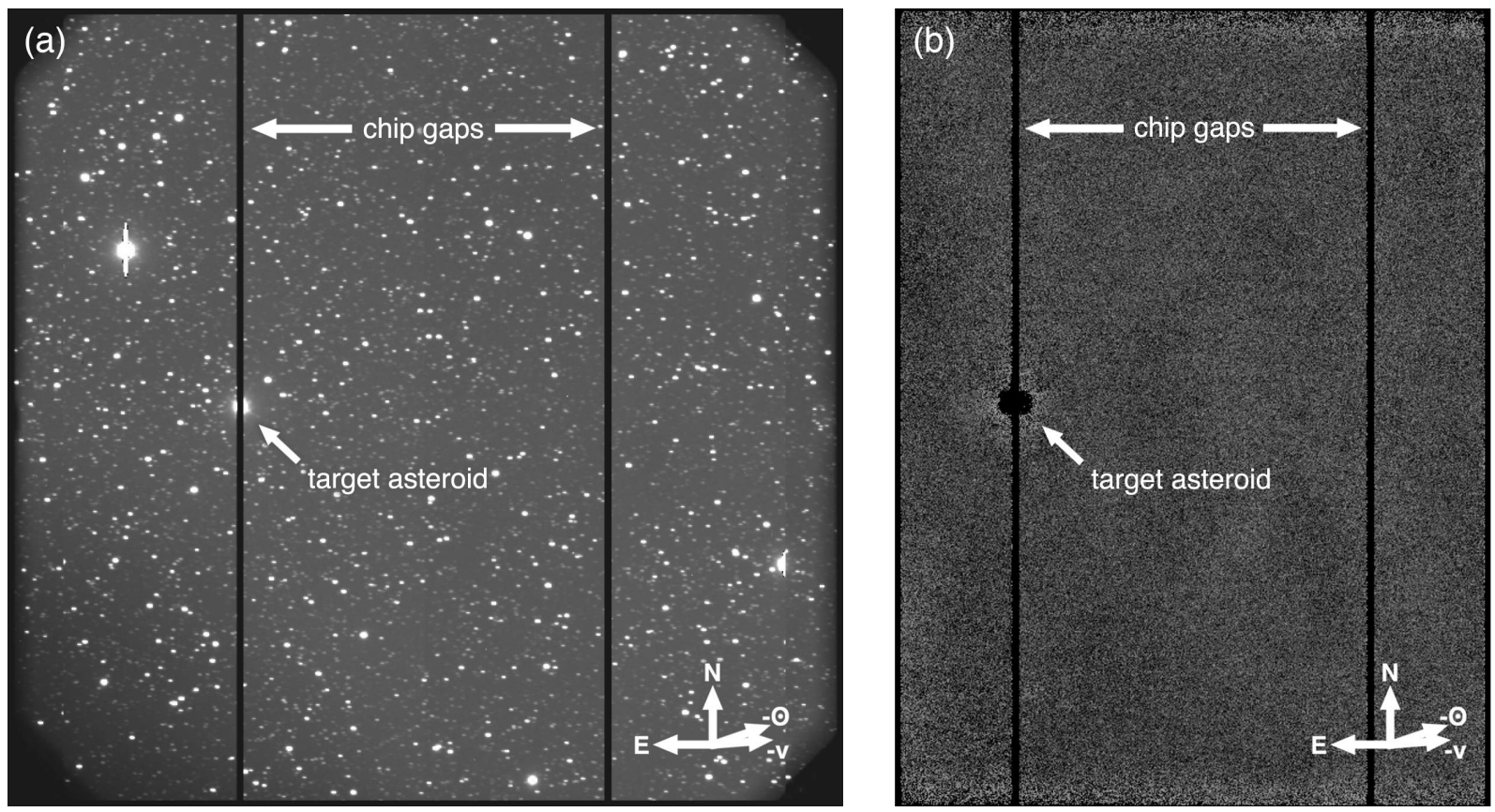

Figure 1. (a) A single 180 s exposure of (24) Themis from Gemini-N on 2015 June 9 showing the positions of the chip gaps and the asteroid. (b) Cleaned composite image (where field stars have been removed) constructed from all data listed in Table 1 ( $2 \mathrm{hrs}$ of total effective exposure time). The locations of the target asteroid and the chip gaps, north (N), east (E), the antisolar direction $(-\odot)$, and the negative heliocentric velocity vector $(-v)$, as projected on the sky, are marked in both panels. The central chip area in both panels is 2.4 wide and 5.0 high.

Table 1. Observation $\log$

\begin{tabular}{lcccccccccrrrr}
\hline \hline \multicolumn{1}{c}{ UT Date } & Telescope & $N^{a}$ & $t^{b}$ & $\mathrm{am}_{\text {mid }}{ }^{c}$ & $\theta_{s}{ }^{d}$ & $\nu^{e}$ & $R^{f}$ & $\Delta^{g}$ & $\alpha^{h}$ & $\mathrm{PA}_{-\odot} \odot^{i}$ & $\mathrm{PA}_{-v}{ }^{j}$ & $\Delta t^{k}$ \\
\hline 2013 October 31 & Perihelion & $\ldots$ & $\ldots$ & $\ldots$ & $\ldots$ & 0.0 & 2.740 & 3.146 & 17.8 & 292.6 & 292.7 & 0 \\
2015 June 09 & Gemini & 20 & 3600 & 1.408 & 0.8 & 117.3 & 3.275 & 2.261 & 0.9 & 289.0 & 274.5 & 586 \\
2015 June 12 & Gemini & 20 & 3600 & 1.481 & 0.7 & 117.8 & 3.278 & 2.263 & 0.3 & 46.9 & 274.7 & 589 \\
\hline \hline
\end{tabular}

${ }^{a}$ Number of exposures.

${ }^{b}$ Total exposure time, in seconds.

${ }^{c}$ Airmass at midpoint of observations.

${ }^{d}$ FWHM seeing, in arcsec.

$e$ True anomaly, in degrees.

${ }^{f}$ Heliocentric distance, in AU.

${ }^{g}$ Geocentric distance, in AU.

${ }^{h}$ Solar phase angle, in degrees.

${ }^{i}$ Position angle of the antisolar vector, in degrees east of north.

${ }^{j}$ Position angle of the negative heliocentric velocity vector, in degrees east of north.

${ }^{k}$ Days past perihelion.

nier et al. 2013). To enable precise positional offsetting of our images for constructing composite images, we derived independent astrometric solutions for each image using the 2MASS point source catalog (Cutri et al. 2003), ultimately attaining an astrometric precision of $0.2^{\prime \prime}$ for each image.

During our observations, Themis was passing through a dense star field near the galactic plane (galactic lat- itude of $b \sim 8^{\circ}$ ), meaning that careful data reduction was needed to search for potential dust emission features against this crowded background. As such, we first masked data within $15^{\prime \prime}$ of Themis's expected position in each snapshot in order to avoid excess flux from the asteroid, and then created a star-aligned composite image excluding masked pixels and CCD gaps. We then used the Hotpants image subtraction software package 
(Becker 2015) ${ }^{3}$ and WCSTools/remap astrometric alignment software (Mink 2011) ${ }^{4}$ to align and resample, convolve, and normalize the resulting background source template to match each individual image for optimal image subtraction, and subtracted each customized template from individual images. For further image refinement, we applied masks to saturated pixels and misaligned remnants in individual subtracted images, using SExtractor's "check image" (Bertin \& Arnouts 1996) as a mask image (after careful visual inspection) to remove all sources except the sky background and any faint dust structures. Individual subtracted images from both nights (comprising a total of two hours of on-source time) were then shifted and aligned (based on Themis's predicted non-sidereal motion on the sky), and medianaveraged to make a new background-subtracted composite image for our search for faint dust structures.

From a visual inspection of our final composite image including data from both nights (Figure 1b), we see no sign of extended dust emission associated with Themis (apart from some residual scattered light from the asteroid itself) down to a $3-\sigma$ limiting magnitude of $\Sigma_{\lim } \sim 28.0 \mathrm{mag} \operatorname{arcsec}^{-2}$, as measured over $1 \operatorname{arcsec}^{2}$. To perform a more quantitative search for a dust trail aligned with Themis's orbit plane as projected on the sky, as was seen for 238P and 324P (Hsieh et al. 2009; Hsieh 2014) and predicted for Themis using syndynesynchrone dust modeling analysis (after Finson \& Probstein 1968), we first rotate our final composite image of Themis to make the expected direction of the trail horizontal in the image frame and then measure the surface brightness of the image within a $3^{\prime \prime}$-wide strip extending to the west (i.e., in the expected direction of the dust trail) using $5^{\prime \prime}$-long rectangular apertures. We also perform the same measurements for strips of sky $10^{\prime \prime}$ North and South of the expected trail in order to measure nearby sky background levels. A diagram of these regions overlaid on our rotated composite image of Themis is shown in Figure 2.

We then calculate the average of the fluxes measured in the two apertures above and below each trail aperture, and subtract the resulting average local sky brightness from the flux measured in each trail aperture to obtain net fluxes along the expected extent of the trail. We plot these calculated net fluxes (in counts per pixel within each $3^{\prime \prime} \times 5^{\prime \prime}$ aperture) in Figure $3 \mathrm{a}$, and equivalent surface brightnesses in magnitudes per arcsec in Figure 3b, where net fluxes less than 3- $\sigma$ above the sky

\footnotetext{
${ }^{3}$ https://github.com/acbecker/hotpants

${ }^{4}$ http://tdc-www.harvard.edu/wcstools/remap.html
}

are assigned magnitudes equal to the limiting magnitude of our observations (as computed over a $3^{\prime \prime} \times 5^{\prime \prime}$ area). From this analysis, we find no clear evidence of excess flux along the direction of the expected trail up to $\sim 2^{\prime}$ from our target object down to a limiting magnitude of $\Sigma_{\lim }=29.7 \mathrm{mag} \operatorname{arcsec}^{-2}$. For comparison, the dust trail detected for MBC $324 \mathrm{P} /$ La Sagra at a true anomaly of $\nu=117^{\circ}$ in 2011 by the Gemini North telescope was seen to extend $>2^{\prime}$ from the comet's nucleus and had a measured surface brightness of $\Sigma_{R} \sim 27.2 \mathrm{mag} \operatorname{arcsec}^{-2}$ (Hsieh 2014).

\section{DISCUSSION}

\subsection{Dust Ejection Physics}

Themis has a radius of $r_{n}=91.9 \pm 5.7 \mathrm{~km}$ and an estimated mass of $M=(5.9 \pm 1.9) \times 10^{18} \mathrm{~kg}$ (Carry 2012). Using

$$
v_{\mathrm{esc}}=\sqrt{\frac{2 G M}{r_{n}}}
$$

to compute the escape velocity of the body, we find an escape velocity of $v_{\text {esc }} \sim 90 \mathrm{~m} \mathrm{~s}^{-1}$. This is extremely high compared to the dust ejection velocities determined for other MBCs (on the order of meters per second; e.g., Hsieh et al. 2004, 2009, 2011a; Moreno et al. 2011, 2013; Licandro et al. 2013) for grain sizes ranging from $\mu \mathrm{m}$ to cm scales.

The relationship between particle radius, $a$, and ejection velocity, $v_{e j}$, typically assumed in numerical comet dust ejection models is given by

$$
v_{e j}(\beta) \approx v_{0} \beta^{1 / 2}
$$

where $\beta$ is the dimensionless ratio of the acceleration due to solar radiation pressure to the local acceleration due to gravity for a particle of a given radius (where $\beta \approx 1 / a$ for $a$ in $\mu \mathrm{m})$ and $v_{0}$ is the reference ejection velocity for a particle with $a=1 \mu \mathrm{m}$ (e.g., Lisse et al. 1998; Reach et al. 2000; Ishiguro et al. 2007). Assuming $v_{0} \sim 25 \mathrm{~m} \mathrm{~s}^{-1}$ as determined for both MBCs $238 \mathrm{P} /$ Read and 324P/La Sagra (Hsieh et al. 2009; Moreno et al. 2011), which gives some of the highest ejection velocities determined for a $\mathrm{MBC}$, we find that only dust particles with $\beta>10$ (cf. Figure 4), corresponding to $a<0.1 \mu \mathrm{m}$, would be expected to reach ejection velocities sufficient to escape Themis's gravity.

Sub- $\mu \mathrm{m}$ particles are not expected to produce the type of long-lasting dust tail that we were searching for with these observations. Given the large size of Themis, however, higher rates of sublimation than any of the MBCs known to date could be possible in principle, given the possibility of larger subsurface volatile reservoirs. For example, as mentioned above, (1) Ceres (with a diameter of $\sim 950 \mathrm{~km}$ ) was observed by Herschel to exhibit a 


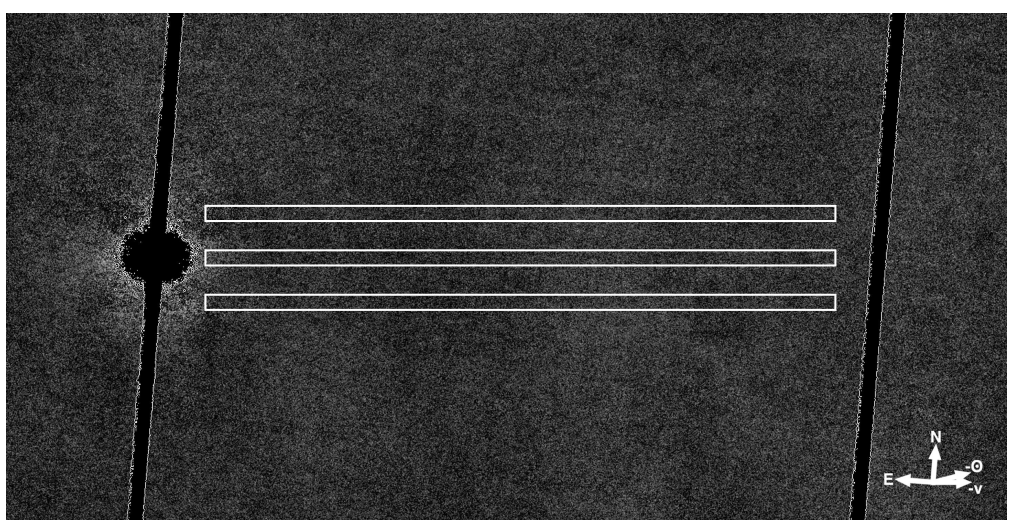

Figure 2. Diagram of the positions of the region of sky used to search for dust emission along the expected direction of Themis's dust trail (middle white rectangle) and the regions used for sky background subtraction (top and bottom white rectangles) overlaid on a rotated star-subtracted composite image of our Themis observations. Themis was located at the center of the large black circle to the left of the image. North $(\mathrm{N})$, east $(\mathrm{E})$, the antisolar direction $(-\odot)$, and the negative heliocentric velocity vector $(-v)$, as projected on the sky, are marked as labeled.
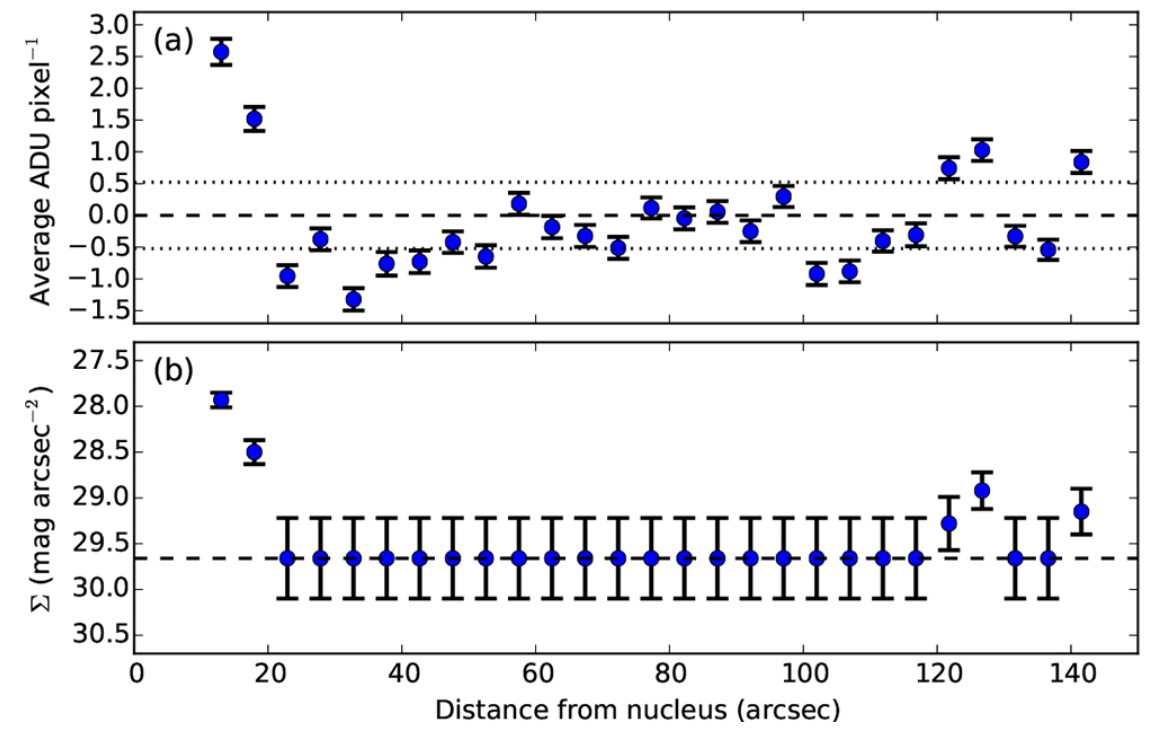

Figure 3. (a) Plot of net flux per pixel (in ADU) for $5^{\prime \prime}$-long apertures along a $3^{\prime \prime}$-wide strip placed along the expected extent of the Themis dust trail in the composite image constructed from data from both nights of observations (schematically shown in Figure 2). Error bars indicating 1- $\sigma$ uncertainties are shown, while points contained within the dotted lines are within 3- $\sigma$ of the sky level. (b) Plot of equivalent surface brightnesses in magnitudes per $\operatorname{arcsec}^{2}$ for the aperture fluxes in (a), where magnitudes for average fluxes less than 3- $\sigma$ above the sky are assigned to the 3- $\sigma$ limiting magnitude of $29.7 \mathrm{mag} \operatorname{arcsec}^{-2}$ (as measured over a $3^{\prime \prime} \times 5^{\prime \prime}$ area) for our observations.

water vapor production rate of $Q_{\mathrm{H}_{2} \mathrm{O}}>10^{26} \mathrm{~mol} \mathrm{~s}^{-1}$, higher than any of the upper limits measured or inferred for known MBCs (e.g., Jewitt et al. 2009, 2015a; Licandro et al. 2011, 2013; Hsieh et al. 2012b,c, 2013; de ValBorro et al. 2012; O'Rourke et al. 2013), perhaps due to either sublimation of near-surface ice (which is likewise a plausible explanation for observed MBC activity) or cryovolcanism powered by heating from long-lived radioisotopes in the deep interior of Ceres (Küppers et al. 2014). In the case of Themis itself, water ice is unstable against sublimation at the calculated equilibrium sur- face temperatures of the object, yet water frost has been detected across its surface (Campins et al. 2010). This suggests that the detected frost layer was recently deposited at the time of its observations, and perhaps periodically or continuously replenished by mechanisms like ongoing outgassing from the asteroid's interior and recondensation via cold-trapping (Rivkin \& Emery 2010), in turn, implying the presence of significant subsurface water ice reservoirs.

To get a sense of the upper range of the strength of comet-like sublimation-driven dust emission that we 


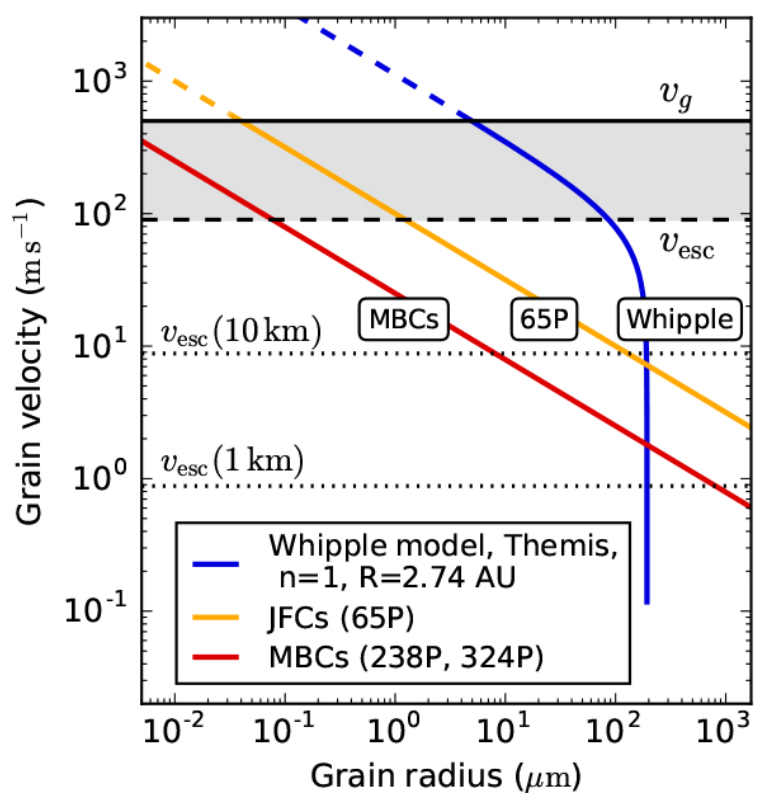

Figure 4. Expected dust grain ejection velocities from Themis as a function of particle size, assuming the Whipple model for dust emission for a "new" classical comet at the heliocentric distance of Themis's perihelion (blue line), the size-velocity relationship for ejected dust grains found for JFC $65 \mathrm{P}$ (orange line; Ishiguro et al. 2007), and the size-velocity relationship for ejected dust grains found for previously analyzed MBCs 238P and 324P (red line; Hsieh et al. 2009; Moreno et al. 2011). A horizontal black solid line marks the assumed mean gas velocity $\left(v_{g}=500 \mathrm{~m} \mathrm{~s}^{-1}\right)$, while a horizontal black dashed line marks the escape velocity for Themis $\left(v_{e s c}=90 \mathrm{~m} \mathrm{~s}^{-1}\right)$ and horizontal black dotted lines mark escape velocities for objects with Themislike densities $\left(\rho=1400 \mathrm{~kg} \mathrm{~m}^{-3}\right)$ and radii of $r=1 \mathrm{~km}$ $\left(v_{\text {esc }}=0.88 \mathrm{~m} \mathrm{~s}^{-1}\right)$ and $r=10 \mathrm{~km}\left(v_{\text {esc }}=8.8 \mathrm{~m} \mathrm{~s}^{-1}\right)$, as labeled. The shaded region indicates the range of velocities over which dust particles exceed Themis's escape velocity, and are therefore ejected and become potentially observable. Velocities above this range are unphysical because $v_{g}$ sets an effective maximum velocity for dust particles ejected by gas drag, while particles with velocities below this range cannot escape Themis's gravity and thus never become observable.

might expect from Themis, we consider the Whipple model (Whipple 1950, 1951) for dust emission in classical comets (i.e., from the outer solar system). In this model, which assumes that sublimation occurs over the entirety of an object's sunlit hemisphere, terminal dust grain velocities are given by

$$
v_{\infty}=\left(\frac{13 v_{g} F}{12 n R[\mathrm{AU}]^{2} a \rho_{d} H} r_{n}-\frac{8 \pi G}{3} \rho_{n} r_{n}^{2}\right)^{1 / 2}
$$

where $F=1361 \mathrm{~W} \mathrm{~m}^{-2}$ is the solar constant, $1 / n$ is the efficiency of solar radiation at sublimating cometary ices, $G=6.67 \times 10^{-11} \mathrm{~m}^{3} \mathrm{~kg}^{-1} \mathrm{~s}^{-2}$ is the gravitational constant, and $r_{n}$ is the radius of central body. Assum- ing a mean gas velocity of $v_{g}=500 \mathrm{~m} \mathrm{~s}^{-1}$, a bulk asteroid density of $\rho_{n}=1400 \mathrm{~kg} \mathrm{~m}^{-3}$ (characteristic of C-type asteroids like Themis; Britt et al. 2002), dust grain densities of $\rho_{d}=2500 \mathrm{~kg} \mathrm{~m}^{-3}$ (characteristic of $\mathrm{CI}$ and $\mathrm{CM}$ carbonaceous chondrite meteorites which are associated with C-type asteroids; Britt et al. 2002), and using Themis's heliocentric distance at perihelion of $R_{q}=2.74 \mathrm{AU}$, and a latent heat of sublimation of $H=2.83 \times 10^{6} \mathrm{~J} \mathrm{~kg}^{-1}$, as appropriate for water ice, we obtain

$$
v_{\infty}=\left(1.27 \frac{1}{n a}-6.6 \times 10^{3}\right)^{1 / 2}
$$

We plot this function along with the size-velocity relationship determined for 238P and 324P (Equation 2) in Figure 4. The Whipple model consistently produces larger dust grain velocities than computed for previously observed MBCs, but also includes a steep drop-off in terminal velocity as a critical dust radius is reached.

Setting $v_{\infty}=0 \mathrm{~m} \mathrm{~s}^{-1}$, we can express the critical dust particle size above which dust particles are too large to be ejected from a Themis-like asteroid by gas drag as

$$
a_{c}=\frac{133.2}{R[\mathrm{AU}]^{2} r_{n}} \frac{1}{n} \approx \frac{200}{n} \mu \mathrm{m}
$$

For a "new" comet (i.e., $n=1$ ), only particles with $a<200 \mu \mathrm{m}\left(\beta>5 \times 10^{-3}\right)$ can be ejected. This result reflects the maximum activity strength that we would expect as a result of sublimation across the entire surface of Themis, where $a_{c}$ will decrease for lower sublimation efficiencies (i.e., larger $n$ ). For comparison, using values appropriate for (62) Erato $\left(r_{n}=53.5 \mathrm{~km}\right.$; $\left.R_{q}=2.61 \mathrm{AU}\right)$, another large Themis family asteroid, we find $a_{c} \approx 350 n^{-1} \mu \mathrm{m}$ under the Whipple model.

Applying simple Finson-Probstein modeling (Finson $\&$ Probstein 1968), we find that $200 \mu \mathrm{m}$ particles ejected at the time of Themis's perihelion passage would be $\sim 1$ degree from the nucleus 590 days later (at the time of our observations), and outside our field of view. To still be visible within 2 arcmin of the asteroid at the time of our observations, $200 \mu \mathrm{m}$ particles would have needed to have been ejected fewer than 200 days prior to our observations, or around November 2014, when Themis was at $R=3.03 \mathrm{AU}$ and $\nu \sim 80^{\circ}$, or later. Compared to the theoretical maximum ejectable particle size for Themis at perihelion $\left(\nu \sim 0^{\circ}\right), a_{c}$ at $\nu \sim 80^{\circ}$ under the full-strength Whipple model is slightly reduced to $a_{c} \sim 160 \mu \mathrm{m}$ (an essentially negligible difference given the expected real-world precision of the critical particle size calculations presented here). However, the sublimation rate of water is roughly an order of magnitude lower at this distance (cf. Hsieh et al. 2015), reducing the likelihood of observable levels of dust emission. 
To summarize, we have considered the case here of the largest ejectable particles from Themis at perihelion assuming that these particles would be the most likely to contribute to the production of a long-lived dust trail. Smaller particles could also produce visible dust emission, but only if they were ejected relatively recently with respect to the time of observations. In the case of the observations presented here, the late timing of our observations relative to Themis's perihelion passage means that any still-visible particles would have had to have been ejected at larger heliocentric distances, at which the sublimation rate of water may have been too low to have produced detectable dust emission. If Themis is actively emitting dust at any point in its orbit, future observations performed earlier in its orbit (i.e., closer to perihelion) would be more likely to reveal visible dust features. Similar searches for dust emission associated with other large, potentially icy asteroids should also be performed relatively close to perihelion to maximize the likelihood of detecting visible dust.

\subsection{Potential Alternative Applications}

Besides performing additional searches for dust emission from Themis and other large, potentially icy asteroids near perihelion, the approach described here could also be useful for searches for dust emitted from large asteroids as a result of processes other than sublimationdriven dust emission. As mentioned above (Section 1.1), collisional erosion of asteroids could be a significant source of interplanetary dust. An online impact simulator created by K. A. Holsapple ${ }^{5}$ demonstrates that for asteroid sizes between $10 \mathrm{~km}$ and $1000 \mathrm{~km}$, the total mass of ejecta material created by impactors greater than $1 \mathrm{~m}$ in diameter that escape the target asteroid, scaled by the target surface area, monotonically increases with the size of the target asteroid. This result implies that searches for faint dust associated with the largest asteroids in the asteroid belt could potentially be highly fruitful.

Dust with velocities significantly exceeding the escape velocity of Themis will necessarily form a trail of greater physical extent than the narrower trails associated with sublimation-driven emission of large particles by shortperiod comets. Such a mechanism may be required to explain the existence of Type II dust trails detected by the Infrared Astronomical Satellite (Sykes 1988), which are more than an order of magnitude wider because of the corresponding greater dispersion in ejection velocities. Searches for such a trail associated with Themis

\footnotetext{
5 http://keith.aa.washington.edu/craterdata/scaling/ index.htm
}

may therefore benefit from the use of facilities with even larger fields of view than GMOS, such as SuprimeCam or HyperSuprimeCam on the Subaru telescope (Miyazaki et al. 2002).

In particular, the approach used in this work could be applied to large asteroids in young asteroid families such as the Veritas and Beagle families (e.g., Milani \& Farinella 1994; Nesvorný \& Vokrouhlický 2006; Nesvorný et al. 2008, 2015; Pravec et al. 2018), to see if dust from intra-family collisional erosion (which may occur at a higher rate for young family members, given the more highly similar orbits of young family members compared to those of other asteroids and their potential impactors) can be detected. While the visibility of ejected dust in these cases is not expected to be necessarily correlated with orbit position (as in the case of sublimation-generated dust emission), consideration of other factors could help to maximize the likelihood of detecting dust features, such as timing observations to coincide with orbit plane crossings (e.g., Hsieh \& Jewitt 2005; Arendt 2014), when the optical depth of a dust trail should be maximized, or close approaches to the Earth, when any dust should simply have a higher apparent surface brightness and thus be more easily detected.

The observational approach presented here could also be used to characterize dust features associated with large, bright asteroids suspected to have experienced recent impacts, such as (596) Scheila or (493) Griseldis (Section 1.1). While the initial detection of dust emission from these objects will usually presumably have been made using more conventional observing techniques, observations similar to those described here could help with the morphological characterization of close-in dust features that would otherwise be impossible to identify amid the large amount of scattered light from such bright objects, or extend the length of time over which fading dust features can be observed following an impact event. Such observations could aid in setting limits on maximum ejected dust grain sizes, total ejected dust masses, and impactor sizes and energies, and also aid in the determination of the cause of the active event itself (e.g., Ishiguro et al. 2011a,b).

\section{SUMMARY AND CONCLUSIONS}

In this paper, we report the results of an attempt to detect sublimation-driven dust emission from the large main-belt asteroid (24) Themis, which has been reported in previous studies to have widespread water ice frost on its surface, and whose collisional family contains at least 3 MBCs. While no dust emission was ultimately detected, we suggest that our observational approach of 
using a chip gap to block much of the light from a bright target asteroid could be employed for future searches for faint dust features associated with Themis or other bright asteroids suspected of potentially exhibiting dust emission for one reason or another.

The observing strategy for the search described here involved searching for an extended dust trail aligned with the orbit plane of the object. Such a dust trail could be relatively long-lasting and, if present, would be expected to extend well past the residual scattered light expected from the $m_{V} \sim 11.5$ mag object being blocked by the GMOS chip gap. More detailed consideration of dust ejection physics, however, indicates that the maximum ejectable particles by sublimation at perihelion from an object as large and massive as Themis were unlikely to still be observable in the GMOS field of view at the time of our observations.

Despite the non-detection of a dust trail in this particular work, the results of our data reduction show that our observational and data reduction procedures would have enabled us to detect faint dust emission as close as 20 arcsec from the object (cf. Figure 3), even in a crowded star field. As such, we suggest that similar future searches for dust emission from Themis or other large asteroids could target the immediate vicinity of the central asteroid, where dust particles small enough to exceed the object's escape velocity are expected to be most easily seen. Given that these small particles should be relatively short-lived, in cases where sublimation-driven dust emission is expected, such searches should also be conducted much closer to perihelion than was done in this particular study, i.e., closer to the assumed time of peak dust emission. In cases where dust emission driven by other mechanisms (such as impacts) is expected, conducting searches near perihelion is not necessarily advantageous because such activity is not expected to be correlated with orbit position. In these cases, searches for dust should still be conducted when the visibility of dust features is expected to be highest, such as during orbit plane crossings, during close approaches to the Earth, or following detected disruption events.

\section{ACKNOWLEDGEMENTS}

We thank Gemini support astronomers K. Chiboucas and L. Fuhrman, and queue observers J. Chavez and E. Lee for their assistance in obtaining our observations, and Gemini Observatory, which is operated by the Association of Universities for Research in Astronomy, Inc., under a cooperative agreement with the NSF on behalf of the Gemini partnership: the National Science Foundation (United States), the National Research Council (Canada), CONICYT (Chile), Ministerio de Ciencia, Tecnología e Innovación Productiva (Argentina), and Ministério da Ciência, Tecnologia e Inovação (Brazil), for providing the opportunity to conduct Fast Turnaround program observations (Program GN-2015A-FT-19). We also thank N. Artemieva and D. O'Brien for useful discussion. H.H.H. acknowledges support from NASA Planetary Astronomy grant NNX14AJ38G, NASA Solar System Observations grant NNX16AD68G, and NASA Early Career Fellowship grant 80NSSC18K0193. This work also benefited from support by the International Space Science Institute, Bern, Switzerland, through the hosting and provision of financial support for an international team, led by C. Snodgrass and including $\mathrm{HHH}$ and AF, to discuss the science of MBCs.

\section{Facilities: Gemini-N(GMOS-N)}

Software: IRAF (Tody 1986, 1993), Hotpants (Becker 2015), WCSTools (Mink 2011), SExtractor (Bertin \& Arnouts 1996)

\section{REFERENCES}

Arendt, R. G. 2014, AJ, 148, 135

Becker, A. 2015, HOTPANTS: High Order Transform of PSF ANd Template Subtraction, Astrophysics Source Code Library, Astrophysics Source Code Library, ascl:1504.004

Bertin, E., \& Arnouts, S. 1996, A\&AS, 117, 393

Bodewits, D., Kelley, M. S., Li, J.-Y., et al. 2011, ApJL, 733, L3

Bottke, W. F., Nolan, M. C., Greenberg, R., \& Kolvoord, R. A. 1994, Icarus, 107, 255
Britt, D. T., Yeomans, D., Housen, K., \& Consolmagno, G. 2002, Asteroids III (Tucson, University of Arizona Press), 485-500

Burbine, T. H. 1998, Meteoritics and Planetary Science, 33, 253

Bus, S. J., Bowell, E., \& French, L. M. 1988, IAUC, 4684

Campins, H., Hargrove, K., Pinilla-Alonso, N., et al. 2010, Nature, 464, 1320

Carry, B. 2012, Planet. Space Sci., 73, 98

Carry, B., Dumas, C., Fulchignoni, M., et al. 2008, A\&A, 478,235 
Cikota, S., Ortiz, J. L., Cikota, A., Morales, N., \&

Tancredi, G. 2014, A\&A, 562, A94

Combe, J.-P., McCord, T. B., Tosi, F., et al. 2016, Science, 353 , aaf3010

Cutri, R. M., Skrutskie, M. F., van Dyk, S., et al. 2003,

VizieR Online Data Catalog, 2246

de Val-Borro, M., Rezac, L., Hartogh, P., et al. 2012, A\&A, 546, L4

Dermott, S. F., Nicholson, P. D., Burns, J. A., \& Houck,

J. R. 1984, Nature, 312, 505

Diego, F. 1985, PASP, 97, 1209

Fernández, Y. R., Jewitt, D. C., \& Sheppard, S. S. 2001, ApJL, 553, L197

—. 2005, AJ, 130, 308

Finson, M. J., \& Probstein, R. F. 1968, ApJ, 154, 327

Hargrove, K. D., Emery, J. P., Campins, H., \& Kelley, M. S. P. 2015, Icarus, 254, 150

Hiroi, T., Zolensky, M. E., Pieters, C. M., \& Lipschutz, M. E. 1996, Meteoritics and Planetary Science, 31, 321

Hook, I. M., Jørgensen, I., Allington-Smith, J. R., et al. 2004, PASP, 116, 425

Hsieh, H. H. 2014, Icarus, 243, 16

Hsieh, H. H., Ishiguro, M., Lacerda, P., \& Jewitt, D. 2011a, AJ, 142, 29

Hsieh, H. H., \& Jewitt, D. 2005, ApJ, 624, 1093

—. 2006, Science, 312, 561

Hsieh, H. H., Jewitt, D., \& Ishiguro, M. 2009, AJ, 137, 157

Hsieh, H. H., Jewitt, D. C., \& Fernández, Y. R. 2004, AJ, 127, 2997

Hsieh, H. H., Meech, K. J., \& Pittichová, J. 2011b, ApJL, 736, L18

Hsieh, H. H., \& Sheppard, S. S. 2015, MNRAS, 454, L81

Hsieh, H. H., Yang, B., \& Haghighipour, N. 2012a, ApJ, 744,9

Hsieh, H. H., Yang, B., Haghighipour, N., et al. 2012b, ApJL, 748, L15

-. 2012c, AJ, 143, 104

Hsieh, H. H., Kaluna, H. M., Novaković, B., et al. 2013, ApJL, 771, L1

Hsieh, H. H., Denneau, L., Wainscoat, R. J., et al. 2015, Icarus, 248, 289

Ishiguro, M., Sarugaku, Y., Ueno, M., et al. 2007, Icarus, 189,169

Ishiguro, M., Hanayama, H., Hasegawa, S., et al. 2011a, ApJL, 741, L24

—. 2011b, ApJL, 740, L11

Jewitt, D., Agarwal, J., Peixinho, N., et al. 2015a, AJ, 149, 81

Jewitt, D., \& Guilbert-Lepoutre, A. 2012, AJ, 143, 21
Jewitt, D., Hsieh, H., \& Agarwal, J. 2015b, Asteroids IV

(Tucson, University of Arizona Press), 221-241

Jewitt, D., Weaver, H., Mutchler, M., Larson, S., \& Agarwal, J. 2011, ApJL, 733, L4

Jewitt, D., Yang, B., \& Haghighipour, N. 2009, AJ, 137, 4313

Keil, K. 2000, Planet. Space Sci., 48, 887

Kresák, L. 1972, in IAU Symposium, Vol. 45, IAU Symposium: The Motion, Evolution of Orbits, and Origin of Comets, 503

Küppers, M., O’Rourke, L., Bockelée-Morvan, D., et al. 2014, Nature, 505, 525

Lebofsky, L. A., Feierberg, M. A., Tokunaga, A. T., Larson, H. P., \& Johnson, J. R. 1981, Icarus, 48, 453

Licandro, J., Alvarez-Candal, A., de León, J., et al. 2008, A\&A, 481, 861

Licandro, J., Campins, H., Tozzi, G. P., et al. 2011, A\&A, 532, A65

Licandro, J., de León, J., Pinilla, N., \& Serra-Ricart, M. 2006, Advances in Space Research, 38, 1991

Licandro, J., Moreno, F., de León, J., et al. 2013, A\&A, 550, A17

Lisse, C. M., A'Hearn, M. F., Hauser, M. G., et al. 1998, ApJ, 496, 971

Low, F. J., Young, E., Beintema, D. A., et al. 1984, ApJL, 278, L19

Magnier, E. A., Schlafly, E., Finkbeiner, D., et al. 2013, ApJS, 205, 20

Mainzer, A. K., Bauer, J. M., Cutri, R. M., et al. 2016, NASA Planetary Data System, EAR-A-COMPIL-5-NEOWISEDIAM-V1.0

McKay, A. J., Bodewits, D., \& Li, J.-Y. 2017, Icarus, 286, 308

Milani, A., \& Farinella, P. 1994, Nature, 370, 40

Mink, J. 2011, WCSTools: Image Astrometry Toolkit, Astrophysics Source Code Library, Astrophysics Source Code Library, ascl:1109.015

Miyazaki, S., Komiyama, Y., Sekiguchi, M., et al. 2002, PASJ, 54, 833

Mommert, M., Hora, J. L., Harris, A. W., et al. 2014, ApJ, 781,25

Morbidelli, A., Chambers, J., Lunine, J. I., et al. 2000, Meteoritics and Planetary Science, 35, 1309

Moreno, F., Cabrera-Lavers, A., Vaduvescu, O., Licandro, J., \& Pozuelos, F. 2013, ApJL, 770, L30

Moreno, F., Lara, L. M., Licandro, J., et al. 2011, ApJL, 738, L16

Nesvorný, D., Bottke, W. F., Levison, H. F., \& Dones, L. 2003, ApJ, 591, 486 
Nesvorný, D., Bottke, W. F., Vokrouhlický, D., et al. 2008, ApJL, 679, L143

Nesvorný, D., Brož, M., \& Carruba, V. 2015, Asteroids IV

(Tucson, University of Arizona Press), 297-321

Nesvorný, D., \& Vokrouhlický, D. 2006, AJ, 132, 1950

Nesvorný, D., Vokrouhlický, D., Bottke, W. F., \& Sykes, M. 2006, Icarus, 181, 107

O'Brien, D. P., Izidoro, A., Jacobson, S. A., Raymond, S. N., \& Rubie, D. C. 2018, SSRv, 214, 47

O'Brien, D. P., Morbidelli, A., \& Levison, H. F. 2006, Icarus, 184, 39

O'Rourke, L., Snodgrass, C., de Val-Borro, M., et al. 2013, ApJL, 774, L13

Pravec, P., Fatka, P., Vokrouhlický, D., et al. 2018, Icarus, 304, 110

Raymond, S. N., \& Izidoro, A. 2017, Icarus, 297, 134

Raymond, S. N., Quinn, T., \& Lunine, J. I. 2004, Icarus, 168, 1

Reach, W. T., Kelley, M. S., \& Sykes, M. V. 2007, Icarus, 191, 298

Reach, W. T., Sykes, M. V., Lien, D., \& Davies, J. K. 2000, Icarus, 148, 80

Rivkin, A. S., \& Emery, J. P. 2010, Nature, 464, 1322
Schlafly, E. F., Finkbeiner, D. P., Jurić, M., et al. 2012, ApJ, 756, 158

Snodgrass, C., Agarwal, J., Combi, M., et al. 2017, A\&A Rv, 25, 5

Sykes, M. V. 1988, ApJL, 334, L55

-. 1990, Icarus, 85, 267

Sykes, M. V., \& Walker, R. G. 1992, Icarus, 95, 180

Takir, D., \& Emery, J. P. 2012, Icarus, 219, 641

Tholen, D. J., Hartmann, W. K., Cruikshank, D. P., et al. 1988, IAUC, 4554, 2

Tholen, D. J., Sheppard, S. S., \& Trujillo, C. A. 2015, in AAS/Division for Planetary Sciences Meeting Abstracts, Vol. 47, AAS/Division for Planetary Sciences Meeting Abstracts, 414.03

Tody, D. 1986, in Proc. SPIE, Vol. 627, Instrumentation in Astronomy VI, 733

Tody, D. 1993, in Astronomical Society of the Pacific Conference Series, Vol. 52, Astronomical Data Analysis Software and Systems II, 173

Tonry, J. L., Stubbs, C. W., Lykke, K. R., et al. 2012, ApJ, 750,99

Vernazza, P., Mothé-Diniz, T., Barucci, M. A., et al. 2005, A\&A, 436, 1113

Whipple, F. L. 1950, ApJ, 111, 375

—. 1951, ApJ, 113, 464 